\title{
Development of a dynamic multi-scale, computational model of human hepatic glucose and fructose metabolism
}

\author{
E.M. Maldonado ${ }^{1}$, M.J. Tindall ${ }^{2}$, N.J. Plant ${ }^{1}$, A.M. Kierzek ${ }^{1}$ and J.B. Moore ${ }^{1,3}$ \\ ${ }^{1}$ Faculty of Health and Medical Sciences, University of Surrey, Guildford GU2 7XH, UK, ${ }^{2}$ Department of Mathematics \\ and Statistics and Institute for Cardiovascular and Metabolic Research, University of Reading, Reading RG6 6AX, UK \\ and ${ }^{3}$ School of Food Science and Nutrition, University of Leeds, Leeds LS2 9JT, UK
}

Very high doses of fructose alter human hepatic insulin sensitivity and increase lipogenesis. However, the relevance of these data to population consumption is unclear. The objective of this work is to develop a predictive, multi-scale model of human hepatic monosaccharide transport, signalling and metabolism. This computational model will be used to predict the regulatory and metabolic outcomes to physiological levels of glucose and fructose in healthy and fatty liver.

Utilising quasi steady state Petri nets $(\mathrm{QSSPN})^{(1)}$, the aim of this work is to build a multi-scale model composed of: (i) the HepatoNet $1^{(2)}$ liver-specific genome-scale metabolic network constrained by in vitro flux measurements; (ii) a model of insulin signalling created by integration of published kinetic models; and (iii) prediction of monosaccharide transport and metabolism, and triacylglycerol production. Alongside this, an immortalised hepatocyte cell line, HepG2, was used to provide in vitro data to experimentally validate in silico predictions. Both insulin sensitivity $(n 3-4)$ and sugar in culture medium $(-/+100 \mathrm{nM}$ insulin; $n 3-5)$ were measured and analysed by one- and two-way ANOVA followed by Dunnett's and Sidak's test post hoc, respectively.

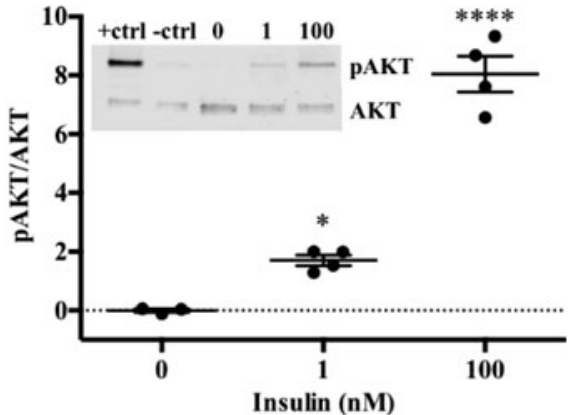

Fig. 1. HepG2 pAKT/AKT.

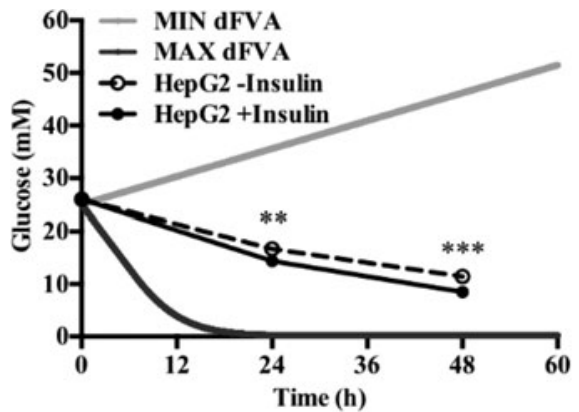

Fig. 2. Extracellular glucose.

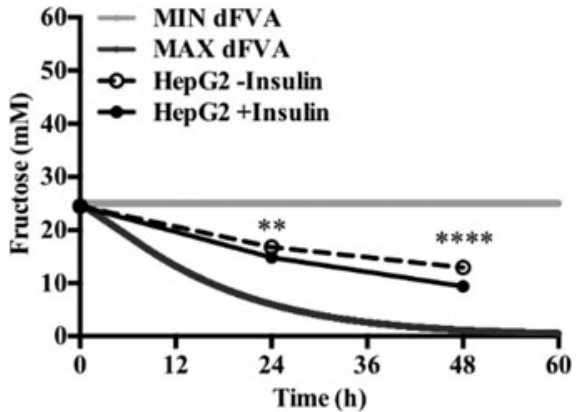

Fig. 3. Extracellular fructose.

To date, we have reconstructed a dynamic regulatory network of hepatic glucose and fructose transport in Petri net formalism and integrated this with HepatoNetl constrained by in vitro data ${ }^{(3)}$. Together with our newly proposed computational analysis approach, 'dynamic flux variability analysis' (dFVA), simulations have predicted minimum and maximum transport rates allowing the calculation of extracellular glucose (Fig. 2) and fructose (Fig. 3) concentrations over time, while also satisfying the demands of a 'healthy hepatocyte'. Insulin sensitivity was confirmed in HepG2 cells with a $1 \cdot 7$-fold increase $(P=0.037)$ of pAKT/AKT expression in response to postprandial levels of insulin (Fig. 1). HepG2 medium glucose and fructose concentrations were found to be within the predicted dFVA range (Fig. 2-3). In addition, a significant increase of sugar uptake was seen in insulin-treated versus untreated cells. Preliminary QSSPN simulations have been successful at replicating the results of a published kinetic model of hepatic insulin signalling ${ }^{(4)}$.

In conclusion, we reproduced in vitro hepatic monosaccharide uptake in our in silico model. Future work will integrate the regulatory insulin signalling network to the metabolic network to predict the outcomes of insulin regulation on sugar and lipid metabolism in response to physiological levels of glucose and fructose in healthy and fatty liver.

1. Fisher CP, Plant NJ, Moore JB, et al. (2013) Bioinformatics 29, 1-9.

2. Gille C, Bölling C, Hoppe A, et al. (2010) Mol Syst Biol 6, 1-12.

3. Jain M, Nilsson R, Sharma S, et al. (2012) Science 336, 1040-44.

4. Kubota H, Noguchi R, Toyoshima Y, et al. (2012) Mol Cell 46, 820-32. 